Ann. Zootech., I980, 29 (3), 3I7-338.

\title{
Utilisation du pâturage par les vaches allaitantes : influence du chargement
}

\author{
M. PETIT $\left({ }^{*}\right)$ et A. MULLER $\left({ }^{* *}\right)$ \\ avec la collaboration technique de D. POURBaix $\left({ }^{*}\right)$, \\ G. PecatTe (**) et Y. Galiard (**) \\ (*) Laboratoive de la Production de Viande, \\ Centre de Recherches de Clermont-Ferrand, I.N.R.A., \\ Theix, 631 Io Beaumont (France) \\ (**) Domaine Expérimental du Pin, I.N.R.A. \\ (Productions Animales) \\ Le Pin-au-Haras, 6rзIo Exmes (France)
}

\begin{abstract}
Résumé
Deux chargements du pâturage ont été comparés de juillet à octobre, au cours de 3 années, avec des vaches normandes allaitant 1,5 ou $\quad$, 7 veau : un chargement normal devant permettre un gain de poids journalier des veaux de $\mathrm{I}$ r oo $\mathrm{g}$ et un chargement fort accru de $30 \mathrm{p}$. I 00 par rapport à la normale. Un $3^{\mathrm{e}}$ traitement consistait en un chargement normal avec 2 veaux par vache ( 2 années) ou un chargement fort dans lequel les veaux recevaient un complément d'aliment concentré ( $\mathrm{I}$ année). Les surfaces expérimentales, précédemment pâturées ou fauchées, ont été exploitées en rotation par les 3 troupeaux qui changeaient de parcelles simultanément.

L'augmentation du chargement (de I,53 à I,97 vache/ha) a réduit la quantité d'herbe offerte par jour et par vache suitée, de $2 \mathrm{I}$ à 32 p. Ioo selon les années. Elle a diminué significativement le gain de poids des veaux de $200 \mathrm{~g}$ en moyenne et n'a pas permis d'accroître le gain de poids de veau à l'hectare. La complémentation en aliment concentré ( $160 \mathrm{~kg} / \mathrm{veau}$ ) a compensé l'effet du chargement élevé. L'accroissement du chargement a, semble-t-il, eu peu d'effet sur la reprise de poids des vaches, mais a parfois réduit significativement leur production laitière.

Pour un même chargement en vaches, l'augmentation du nombre de veaux allaités par vache maximise la production à l'hectare, mais réduit le gain de poids journalier des veaux de $r_{5} \mathrm{~g}$ en raison de la plus faible quantité de lait ingérée par veau et du chargement en veaux plus élevé.

Ces résultats confirment l'importance de la quantité et qualité de l'herbe offerte en été et automne aux veaux de vaches allaitantes. Cependant, les effets du chargement sur la reconstitution des réserves corporelles des vaches sont difficiles à apprécier. De telles études devraient tenir compte des possibilités de restriction du niveau d'alimentation des vaches durant l'hiver suivant.
\end{abstract}




\section{Introduction}

Entretenir un troupeau de vaches allaitantes à l'herbe impose de réduire considérablement le chargement au cours de la saison de pâturage. En effet, la quantité d'herbe volontairement consommée par les veaux augmente quasi linéairement avec l'âge ( $L_{E}$ NEINDre, PETit et MULIER, I976; LE DU, BARker et BAKER, I976). Les besoins du couple mère-veau augmentent donc ou tout au moins restent quasi constants si on tient compte de la reprise de poids des vaches au printemps et de la baisse de production laitière en automne. Dans le même temps, la production fourragère diminue. Ce déséquilibre entre l'évolution des besoins du troupeau et celle de la quantité d'herbe disponible à l'hectare risque d'être encore accru dans le cas de l'allaitement de plusieurs veaux par vache. Une fertilisation azotée importante ne peut d'ailleurs guère y porter remède, puisqu'elle stimule la pousse printanière sans augmenter sensiblement la production automnale.

Les études concernant les effets du chargement au pâturage avec un troupeau de vaches allaitantes restent peu nombreuses et partielles, en comparaison de celles entreprises avec des brebis suitées, principalement en Grande-Bretagne (Gibb et Treacher, I978) et en Australie (Langlands, I977). Drennan (I97I $a$ et $b$ ) a mesuré, dans les conditions irlandaises et au cours de la presque totalité de la période de pâturage, les conséquences sur la croissance des veaux et la variation de poids des vaches d'un accroissement du chargement, soit en vaches suitées, soit setulement en veaux allaités par le truchement de l'allaitement de 2 veaux par vache. HENNESSY et RoBINSON (I979) ont également comparé différents chargements avec des vaches Hereford présentes toute l'année sur les pâturages.

Dans le présent travail, nous avons préféré ne mesurer les conséquences d'un accroissement du chargement qu'à partir du début de l'été, les troupeaux ayant préalablement pâturé avec une pression de pâturage modérée. En effet, non seulement la quantité d'herbe disponible est-elle généralement suffisante au printemps, mais encore les vaches allaitantes doivent à cette époque être fécondées et reconstituer leurs réserves corporelles utilisées au cours de la période hivernale précédente. Les chargements étudiés ont différé, soit par le nombre de vaches allaitant leurs veaux, soit seulement par le nombre de veaux allaités par vache, comme dans les travaux de DRENNAN. Dans le cas d'un chargement élevé, on a également tenté de compenser la réduction de la quantité d'herbe disponible pour les veaux par un apport complémentaire d'aliment concentré.

\section{Matériel et méthodes}

\section{I. - Traitements expérimentaux}

L'étude s'est déroulée au domaine du Pin-au-Haras, en Normandie, de I 973 à I975. Chaque année ont été comparés 2 chargements en vaches suitées, l'un considéré comme normal $(\mathrm{N})$, tel que la croissance des veaux fût élevée (I Ioo $\mathrm{g} / \mathrm{j}$ environ) tout au cours de la période expérimentale, l'autre fort $(\mathrm{F})$, accru de 3o p. Ioo par rapport au précédent. Il s'agissait de vaches normandes allaitant 


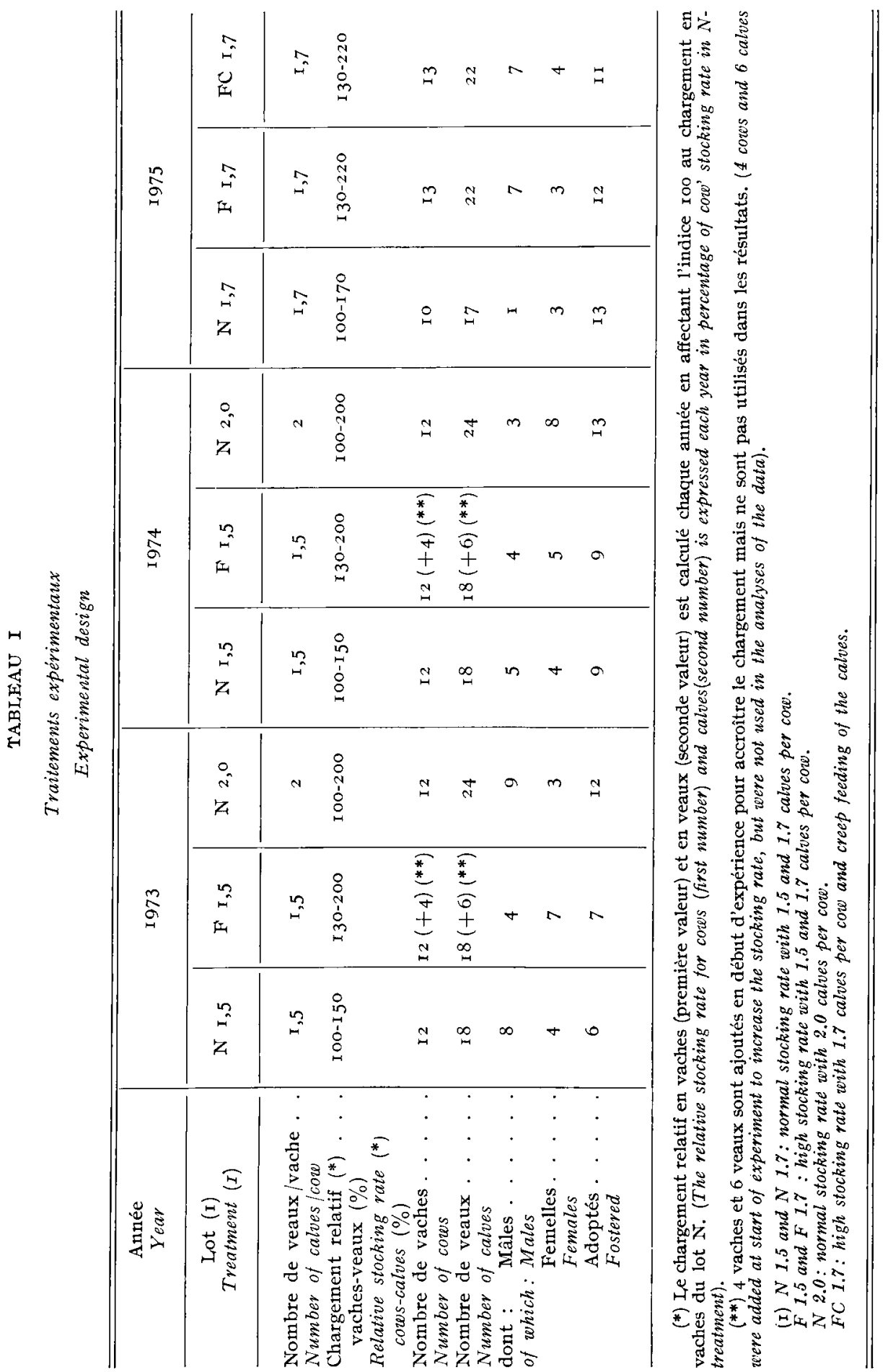


en moyenne I,5 veau en I973 et I 974 ( $\operatorname{lots}$ N I,5 et lots F I,5) et I,7 veau en $\mathrm{I} 975$ (lot $\mathrm{N} \mathrm{I,7}$ et lot $\mathrm{F}$ I,7) (tabl. I).

Les 2 premières années, un $3^{\mathrm{e}}$ lot était conduit avec un chargement normal en vaches mais fort en veaux, chaque vache de ce lot allaitant 2 veaux (lot $N 2,0)$. La dernière année enfin, le $3^{\mathrm{e}}$ lot était conduit en chargement fort avec $\mathrm{I}, 7$ veau par vache, mais les veaux y recevaient un aliment concentré distribué à volonté (lot $\mathrm{FC} I, 7)$. Cette dernière étude n'a pas pu être reconduite en raison des conditions de sécheresse extrêmes de 1976 .

Si l'indice roo est attribué chaque année au chargement normal en vaches, on obtient les chargements relatifs indiqués au tableau I. Il est à noter en particulier que les chargements en veaux sont identiques pour les lots $F$ I,5 et $N_{2}, 0$.

Les 3 troupeaux ont été conduits en rotation et changeaient de parcelles simultanément. Ce sont toujours les lots conduits à chargement le plus faible (lots N) qui ont déterminé les dates de changements de parcelles.

\section{2. - Surfaces expérimentales}

Le domaine du Pin-au-Haras bénéficie d'un climat océanique. I1 est situé sur un sol très argileux et des problèmes de portance des sols limitent la saison de pâturage à 6 mois et demi en moyenne ( $5 / 4$ au I/II). Les conditions climatiques des 3 années expérimentales sont représentées sur la figure $I$.

Au cours des 3 années, les mêmes prairies ont été utilisées ( 23 ha), à savoir I prairie naturelle $(4,56 \mathrm{ha})$ et 2 prairies temporaires de ray-grass anglais, semées en I 968 avec les variétés "Réveille» et "Perma». Lres prairies ont reçu $80 \mathrm{~kg}$ d'acide

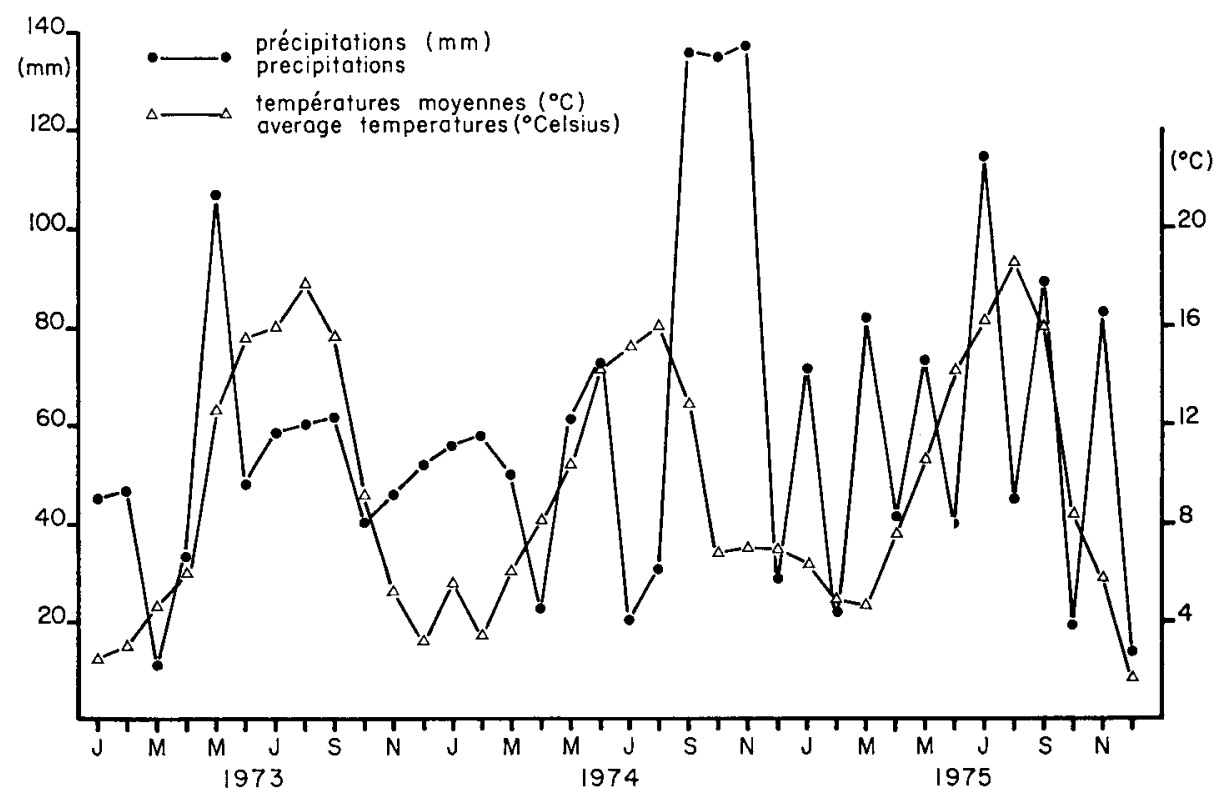

FIG. I. - Températures et précipitations mensuelles.

Monthly temperatures and precipitations. 
phosphorique et de potasse en hiver et $40 \mathrm{~kg}$ d'azote au printemps, ainsi qu'après les $\mathrm{I}^{\mathrm{er}}, 2^{\mathrm{e}}$ et $3^{\mathrm{e}}$ cycles d'exploitation, soit $\mathrm{I} 60 \mathrm{~kg} / \mathrm{ha} / \mathrm{an}$ d'azote. Au printemps, les excédents d'herbe ont été récoltés le plus souvent en ensilage, parfois en foin.

En période expérimentale, on a utilisé des repousses après 2 cycles d'exploitation par pâturage suivi d'une fauche des refus ou des repousses après une fauche. La surface expérimentale totale a été également répartie en 3 surfaces identiques et comprenant 5 (ou 6 en I975) parcelles chacune en tenant compte de la nature des parcelles et de leurs antécédents (productivité au cours des années précédentes, pâturage ou fauche en période pré-expérimentale, âge des repousses). Chaque groupe de parcelles a été attribué au début de l'été à un troupeau expérimental qui y est demeuré jusqu'à la fin de la période de pâturage.

\section{3. - Animaux}

Chaque année, les 3 lots de Io à I 3 vaches expérimentales ont été constitués d'après la date de mise-bas et le poids après vêlage. Les vaches utilisées étaient en $2^{\mathrm{e}}, 3^{\mathrm{e}}$ ou $4^{\mathrm{e}}$ lactation pour la plupart d'entre elles. Elles avaient vêlé en stabulation de la mi-janvier à la fin avril, à une date moyenne toujours située à la fin $\mathrm{du}$ mois de février. L'alimentation hivernale des vaches était identique pour les 3 années : ensilage d'herbe à volonté, foin limité à $3 \mathrm{~kg} / \mathrm{animal} / \mathrm{jour}$, un complément minéral et en lactation un supplément de I à $3 \mathrm{~kg}$ d'aliment concentré selon leur état et le nombre de veaux qu'elles allaitaient. Les tentatives d'adoption de veaux supplémentaires étaient réalisées juste ou peu après le vêlage dans une case individuelle où vaches et veaux restaient durant I semaine (4 à I 2 jours), les tétées étant alors fréquemment surveillées. Les veaux supplémentaires étaient tous des mâles de race normande, pesant 35 à Ioo $\mathrm{kg}(55 \mathrm{~kg}$ en moyenne) lors de la tentative d'adoption; il en était de même des veatux remplaçant les veaux nés dans le troupeau et morts dans le jeune âge, particulièrement nombreux en 1974 et I975 (tabl. I).

La mise à 1'herbe a eu lieu en avril. Durant la période pré-expérimentale de printemps, les 3 troupeaux ont pâturé les parcelles expérimentales mais étaient conduits indépendamment les uns des autres à une pression de pâturage aussi identique que possible. Ils ont été légèrement remaniés peu avant le début de la période expérimentale pour tenir compte de la croissance des veaux depuis leur naissance ou adoption.

\section{4. - Mesures}

Les vaches ont été pesées 3 à 7 jours après le vêlage et I 2 à I7 jours après la mise à l'herbe, I à 4 jours après le début de chaque cycle d'exploitation, à la fin de la période de pâturage et ensuite I à 3 semaines après la rentrée à l'étable. Les pesées étaient effectuées le matin à 9 heures sans jeûne préalable. Leur production laitière a été estimée I à 2 fois par cycle de pâturage, par pesée des veaux avant et après la tétée 2 fois par jour.

L,es veaux on été pesés à la naissance ou à l'adoption et en même temps que les vaches. Les poids des veaux obtenus lors des contrôles laitiers n'ont pas été pris en compte pour 1'estimation de leur gain de poids vif, qui aurait alors été sous-estimé (LE NFindRE, résultats non publiés).

En I974 et I975, on a procédé à des prélèvements d'herbe juste avant l'entrée 
des animaux sur chaque parcelle. Ces prélèvements, d'environ $5 \mathrm{~m}^{2}$ chacun, au nombre de 6 à 12 par parcelle ont été effectués à la motofaucheuse. Par déshydratation à l'étuve d'un échantillon d'environ I $\mathrm{kg}$ de matière verte par prélèvement, on a obtenu une estimation de la matière sèche offerte aux troupeaux.

\section{5. - Traitement des données}

Afin de mettre en évidence d'éventuelles différences de croissance des veaux ou de reprise de poids et production laitière des vaches entre lots, nous avons effectué pour chaque année une série d'analyses statistiques grâce à un modèle d'analyse de variance-covariance pouvant inclure plusieurs facteurs et covariables (SEEBECK, I973).

Ces analyses concernaient :

- le gain de poids journalier des veaux pendant le $3^{\mathrm{e}}$, pendant le $4^{\mathrm{e}}$ $\left(+5^{\mathrm{e}}\right)$ cycle d'exploitation et pendant l'ensemble de la période expérimentale. Les facteurs retenus étaient le lot, l'origine ou le sexe des veaux (o des mères, o des mères, veaux adoptés). La covariable retenue était le poids des veaux en début d'expérience qui incluait à la fois les effets du poids à la naissance ou à l'adoption, de la croissance pré-expérimentale et de l'âge des veaux en début d'expérience;

- la variation totale de poids des vaches en période expérimentale (du début du $3^{\mathrm{e}}$ cycle à la fin du pâturage) et du début de l'expérience jusqu'au début de l'hiver suivant. Ces gains de poids ont été corrigés pour tenir compte du poids des fœtus, des enveloppes et liquides fœtaux (BERESKIN et TouchBERRY, I967) et il a été tenu compte dans l'analyse, du poids des vaches en début d'expérience;

- la production laitière des nourrices en période expérimentale (moyenne de 2 à 3 contrôles laitiers) en utilisant comme covariables la production laitière moyenne en période préexpérimentale (moyenne de 2 à 3 mesures) et le stade de lactation en début d'expérience.

\section{Résultats}

\section{Période pré-expérimentale}

Les dates de vêlage, de mise à l'herbe et de début d'expérience (début du $3^{\mathrm{e}}$ cycle) sont indiquées au tableau 2 . La durée de la période pré-expérimentale au pâturage a différé sensiblement selon les années : les 2 premiers cycles d'exploitation ont été particulièrement courts en 1975 avec un printemps peu favorable à la pousse de l'herbe. Ils ont été à l'inverse beaucoup plus longs en I974.

Pendant la période pré-expérimentale de pâturage, les vaches ont repris $34 \mathrm{~kg}$ de poids vif en 67 jours en moyenne pour les 3 années d'expérience. Les reprises de poids ont été voisines dans les 3 lots en 1973 et I974, bien que les vaches allaitant 2 veaux aient repris la première année moins de poids que celles allaitant $\mathbf{I}, 5$ veau, mais non significativement. En 1975, les vaches du lot F I,7 ont repris significativement $(\mathrm{P}<0,05)$ plus de poids que celles du lot $\mathrm{FC} 1,7$ (tab1. 2).

La production laitière des vaches allaitant 2 veaux a été généralement signi- 


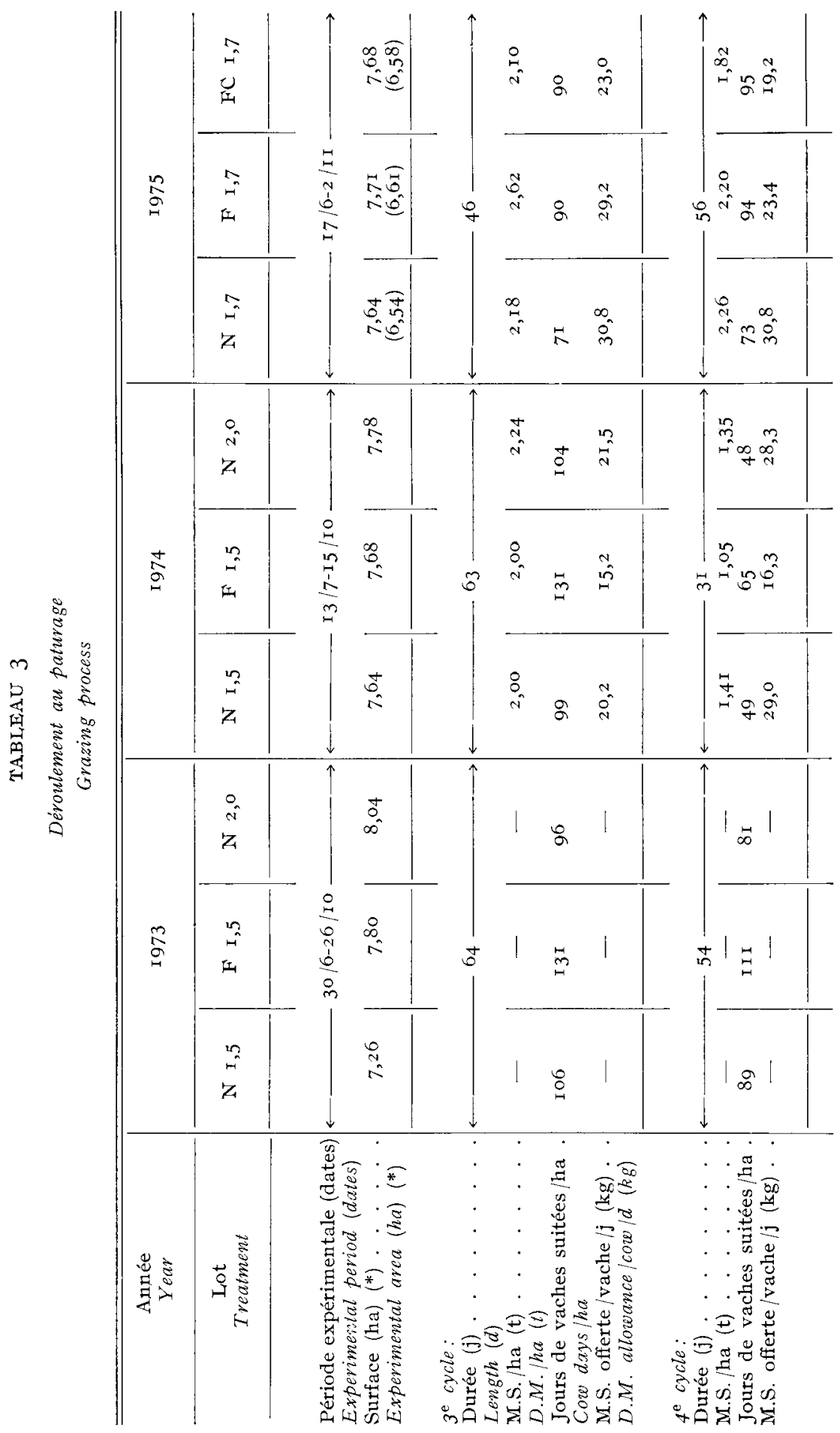




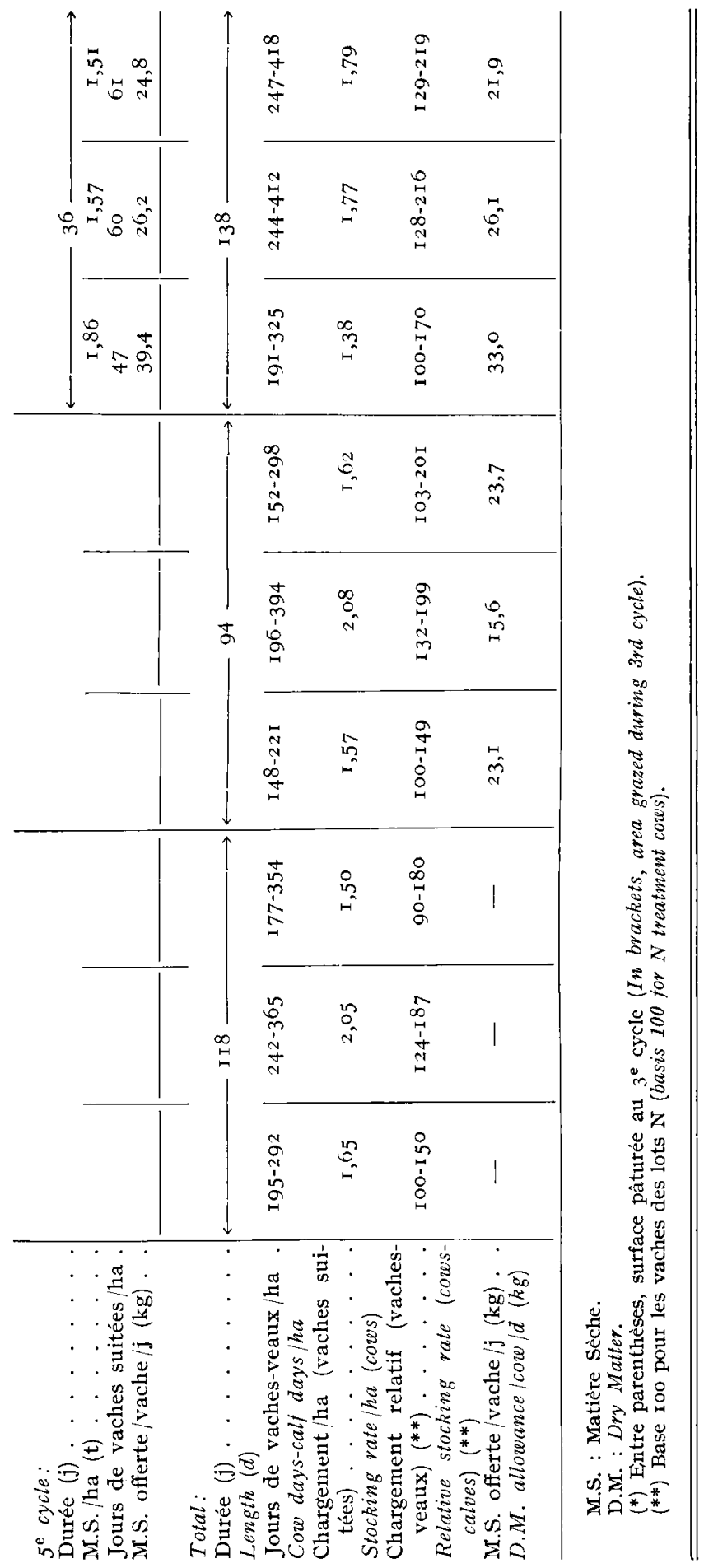


ficativement supérieure au cours de la période pré-expérimentale à celle des vaches allaitant $I, 5$ veau (tabl. 5), ce qui est en accord avec les résultats obtenus par d'autres auteurs (Har'te, I970; Grenet, I974; Pe'tit, Garei, et Le NeINDre, I978).

Les poids et âges des veaux en début d'expérience ont été voisins entre les différents lots chaque année. Les veaux ont été les plus lourds en I974 mais aussı les plus âgés (tab1. 2).

\section{Déroulement du pâturage et quantité d'herbe offerte}

Les périodes expérimentales ont duré I I 8 , 94 et 138 jours, respectivement en 1973,74 et 75 . L'automne froid et pluvieux a écourté la saison de pâturage en 1974 .

I,es chargements sont restés constants pendant toute la période expérimentale, sauf en 1975 où chacun des 3 lots a pâturé à partir du $4^{\text {e }}$ cycle une surface supplémentaire de $I, 1$ ha préalablement fauchée. Les chargements relati $f_{S}$ observés n'ont pas différé sensiblement des chargements prévus, sauf en I973 où le chargement en vaches du lot $\mathrm{N}_{2}, 0$ a été inférieur de Io p. Ioo à celui du lot $\mathrm{N} I, 5$ (tabl. 3).

La quantité de matière sèche d'herbe disponible à l'hectare a été estimée en I 974 et I 975 seulement. Elle était presque équivalente entre les 3 lots au début de la période expérimentale en I974. Mais il semble qu'en I975 les vaches du lot F I,7 aient disposé dès le début de l'expérience de surfaces plus productives que celles des 2 autres lots.

Pour les lots N, la quantité de matière sèche offerte à l'hectare a diminué $\mathrm{du} 3^{\mathrm{e}}$ au dernier cycle de I5 à $30 \mathrm{p}$. Ioo selon l'année, principalement en raison de la diminution normale de la croissance de l'herbe au cours de la saison. Cependant, la quantité de matière sèche offerte par jour et par couple mère-veau a augmenté car le nombre de jours de pâturage à l'hectare a été réduit dans le même temps de $33 \mathrm{p}$. IOO en 1975 et de $50 \mathrm{p}$. IOO en I974.

Au delà $\mathrm{du} 3^{\mathrm{e}}$ cycle, la matière sèche disponible à l'hectare a été la plus faible dans le cas des lots $F$, sans doute en raison des effets cumulatifs du chargement élevé (tabl. 3). Finalement, par jour d'expérience et par couple mère-veau, les animaux des lots $F$ ont disposé de 68 p. Ioo en I974 et 79 p. Ioo en I975 de la matière sèche offerte à ceux des lots $\mathrm{N}$.

\section{Gain de poids vif des vaches}

Le gain de poids vif total des mères en expérience a varié fortement suivant les lots et les années de - $64 \mathrm{~kg}$ ( $\operatorname{lot} \mathrm{N}$ I,7 en I975) à $+4 \mathrm{I} \mathrm{kg}$ ( $\operatorname{lot} \mathrm{N} 2,0$ en I973) (fig. 2). Toutes les vaches ont repris du poids en période expérimentale en I973, surtout au cours du $3^{\mathrm{e}}$ cycle, et en ont perdu en 1974 et $\mathrm{I} 975$. Ces variations annuelles ne semblent pas liées à des évolutions différentes des poids en périodes pré-expérimentales. Toutefois, elles sont atténuées quand on considère les gains de poids jusqu'au début de l'hiver suivant. Le poids vif a peu varié en 1973 lors du passage du pâturage à la stabulation hivernale et a augmenté de 33 et $38 \mathrm{~kg}$ respectivement en $\mathrm{I} 974$ et $\mathrm{I} 975$. Ces variations de poids vif lors de la rentrée à l'étable reflètent en grande partie celles du poids du contenu digestif qui était 


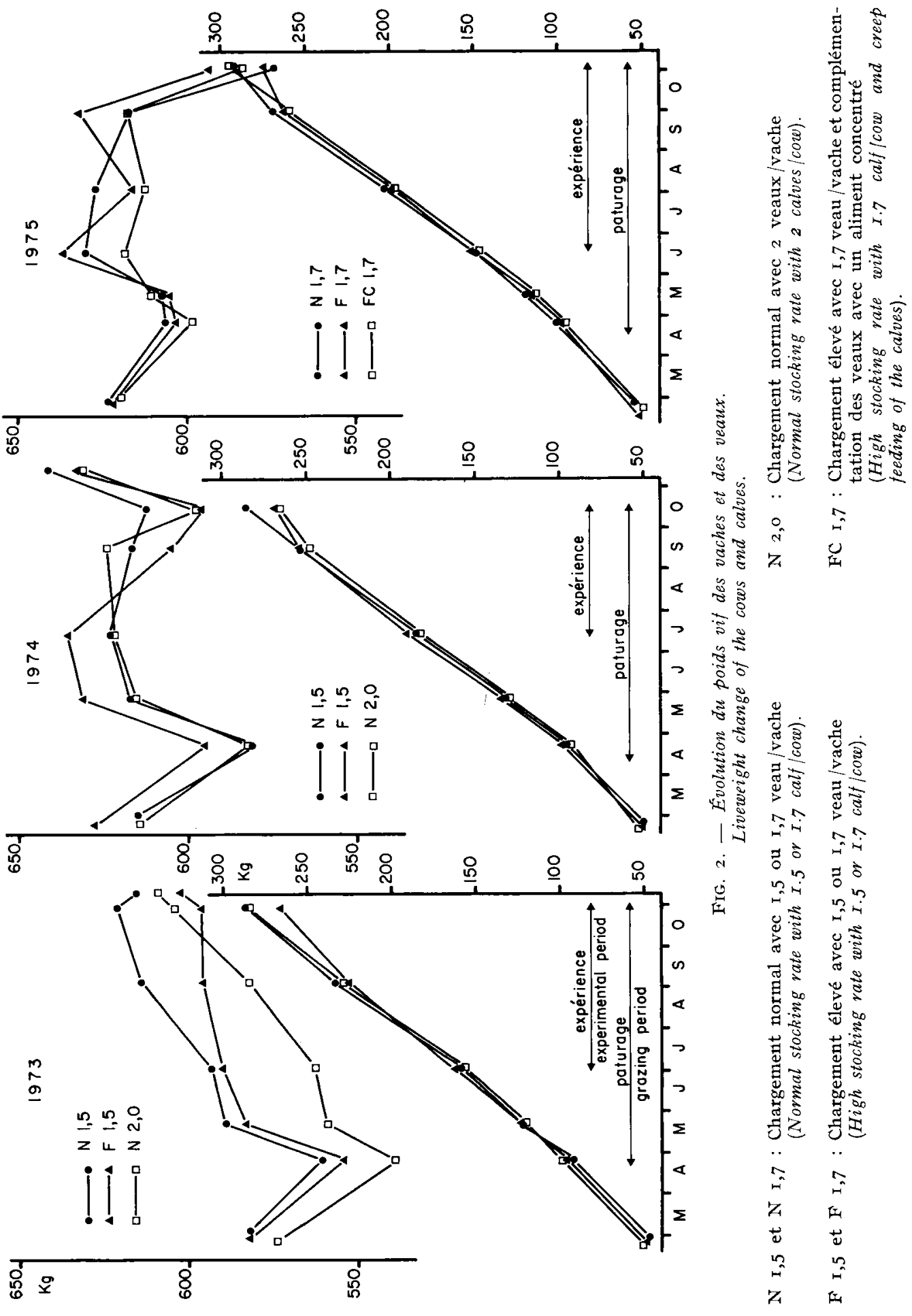


M. PETTT, A. MULLER

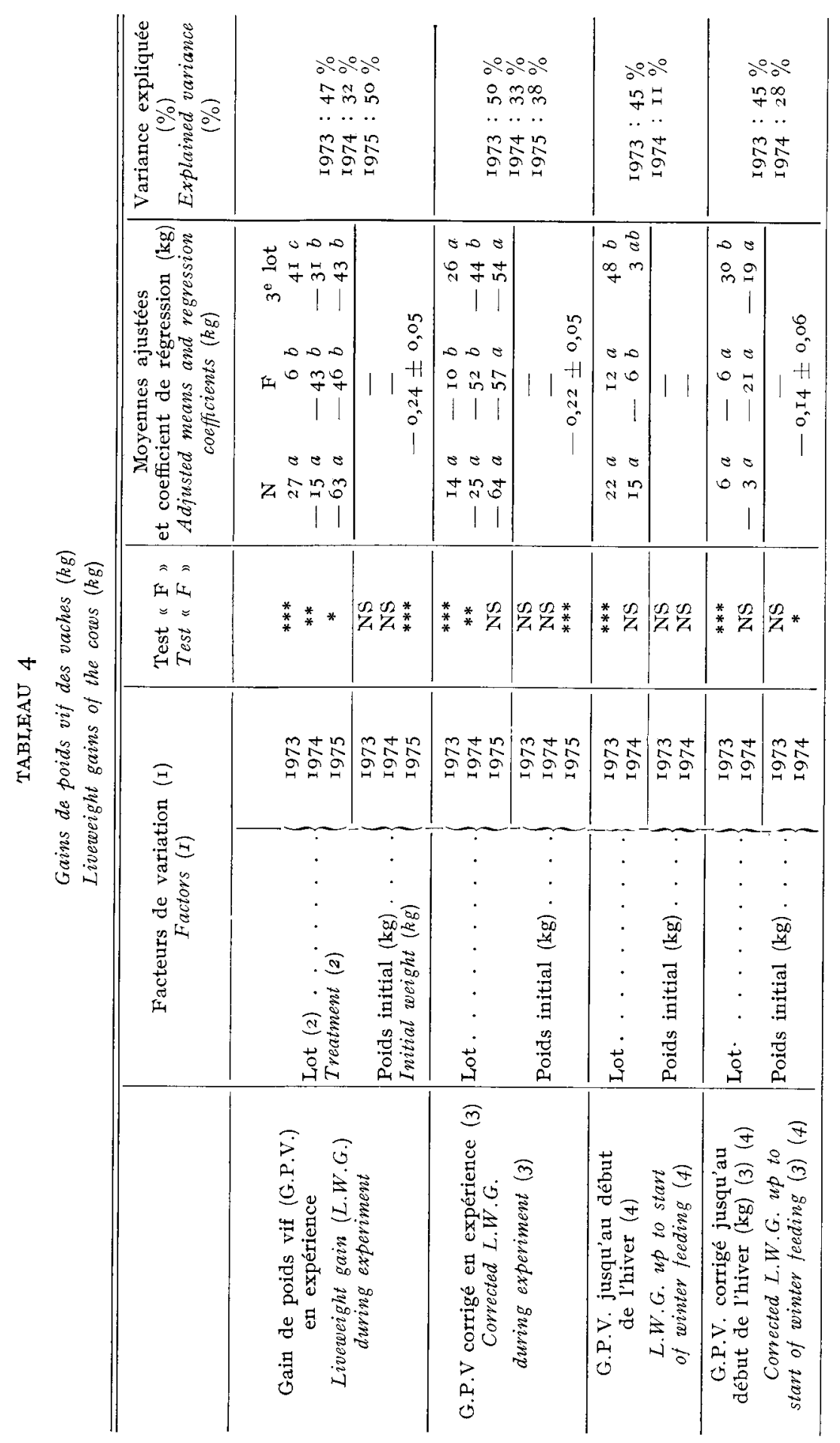

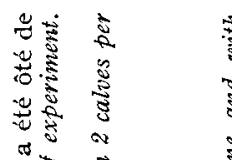

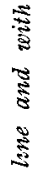

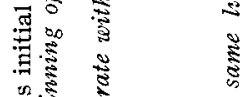

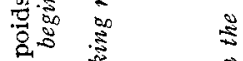

पे

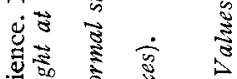

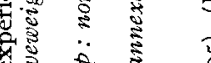

गे है के

$+\frac{7}{2}$ 咅 $V$

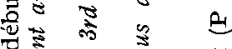

\#

का 0 क

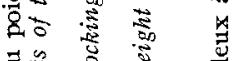

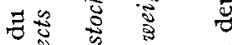

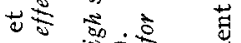

도 출

च.

प्छ छ

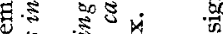

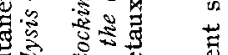

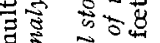

है छี

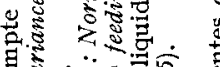

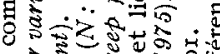

亖

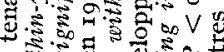

. क म

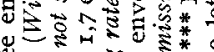

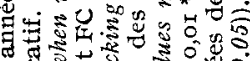

马

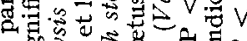

o

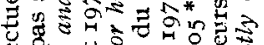

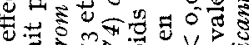

\&:

击

क象。

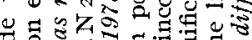

品

\%

可.

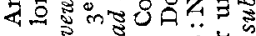

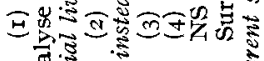
तु. के 
sans doute plus homogène d'une année à l'autre au début de l'hiver qu'à la fin du pâturage.

Les vaches des lots $\mathrm{N}$ ont repris en période expérimentale significativement plus de poids que celles des lots $\mathrm{F}$ les 2 premières années d'expérience : respectivement +27 et $+6 \mathrm{~kg}$ en I973 $(\mathrm{P}<\mathrm{o}$,OI $)$ et $-\mathrm{I} 5$ et $-43 \mathrm{~kg}$ en I974 $(\mathrm{P}<0,0 \mathrm{r})$. L'efiet du chargement est encore sensible après correction des gains de poids pour le poids du fotus et des enveloppes. En revanche, la différence n'est plus significative entre les 2 lots pour la reprise de poids corrigée jusqu'au début de l'hiver (tabl. 4).

En I975, les vaches du chargement normal ont perdu plus de poids que celles des 2 autres lots. Mais cela peut être attribué en grande partie à des différences dans les proportions de vaches pleines : $\operatorname{lot} \mathrm{N}_{\mathrm{I}, 7}=\mathrm{I} / \mathrm{I0} ; \operatorname{lot} \mathrm{F}_{\mathrm{I}, 7}=\mathrm{I} 2 / \mathrm{I} 3$; lot $\mathrm{FC} I, 7=\mathrm{I} 2 / \mathrm{I}_{3}$. Après correction pour les poids des produits de la conception, il n'apparaît plus aucune différence significative.

Les vaches des lots $N_{2}$,o ont eu un comportement très différent en I973 et I974. Flles ont repris jusqu'au début de l'hiver significativement plus de poids que les vaches des 2 autres lots en $1973(\mathrm{P}<0$,oor). Elles ont perdu plus de poids que celles du lot $\mathrm{N}_{\mathrm{I}, 5}$ en $\mathrm{r} 974$.

Le poids des vaches en début d'expérience n'a d'effet significatif sur la reprise de poids ultérieure qu'en I975 ( $\mathrm{P}<0$,00x), les vaches les plus lourdes ayant alors perdu plus de poids par la suite (tak1. 4).

\section{Production laitière}

De la période de pâturage pré-expérimentale à la période expérimentale, la production laitière a chuté significativement pour tous les lots en I973 et 1974 $(\mathrm{P}<\mathrm{o}$,or, test de $t)$, mais pas en $\mathrm{I} 975$.

En période expérimentale, l'augmentation du chargement n'a réduit significativement la production laitière qu'en I974, de $I, 6 \mathrm{~kg} / \mathrm{j}(\mathrm{P}<0$, 01 et $\mathrm{P}<0,05$ respectivement en tenant compte ou pas de la production en pré-expérience) (tabl. 5).

Comme en période pré-expérimentale, les vaches allaitant 2 veaux ont une production laitière significativement supérieure à celle des vaches allaitant $\mathrm{I}, 5$ veau $(\mathrm{P}<0,05$ en $\mathrm{I} 974$ par rapport au lot $\mathrm{N}, \mathrm{P}<0$,oor en 1973 et 1974 par rapport au lot $\mathrm{F}$ ). Cependant, ramenées à une même quantité de lait produite pendant le pâturage de printemps, les productions laitières en période expérimentale ne varient plus avec le nombre de veaux allaités. Enfin, dans chaque lot, les vaches les plus productives au printemps le restent en période expérimentale $(\mathrm{P}<\mathrm{O}, \mathrm{OI})$ et la production laitière ne diminue significativement avec le stade de lactation initial qu'en 1975 (tab1. 5).

\section{Gain de poids des veaux}

L'objectif fixé de I Ioo g/j pour les veaux du lot $\mathrm{N}$ a été approché chaque année, mais avec une assez forte hétérogénéité du gain de poids à l'intérieur du lot : 1'écart-type de gain de poids vif en expérience a varié de I66 g en I973 et 1975 à $20 \mathrm{I} \mathrm{g}$ en I974, soit un coefficient de variation de I6 à I9 p. Ioo.

Le gain de poids journalier pendant la période expérimentale a augmenté 


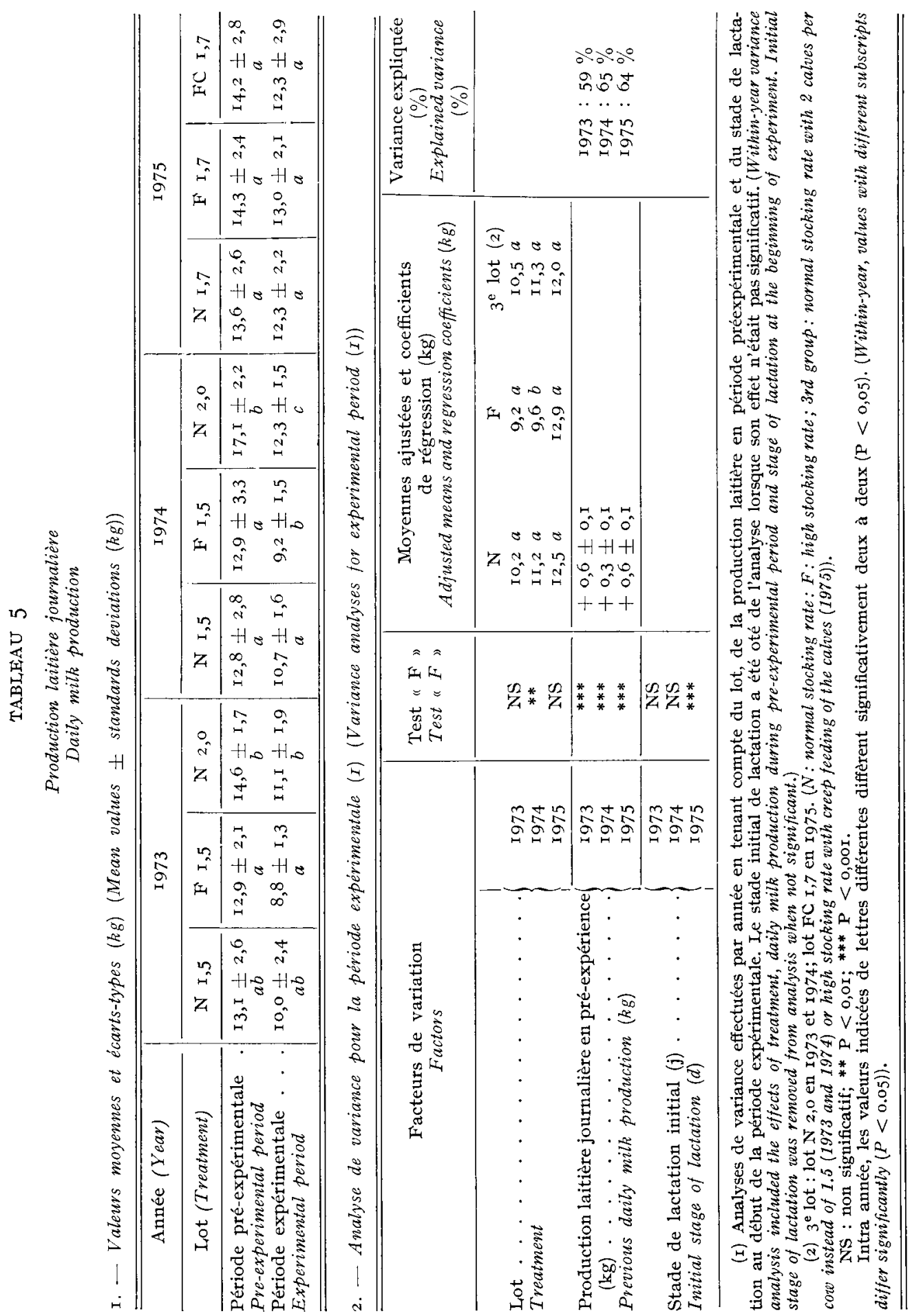




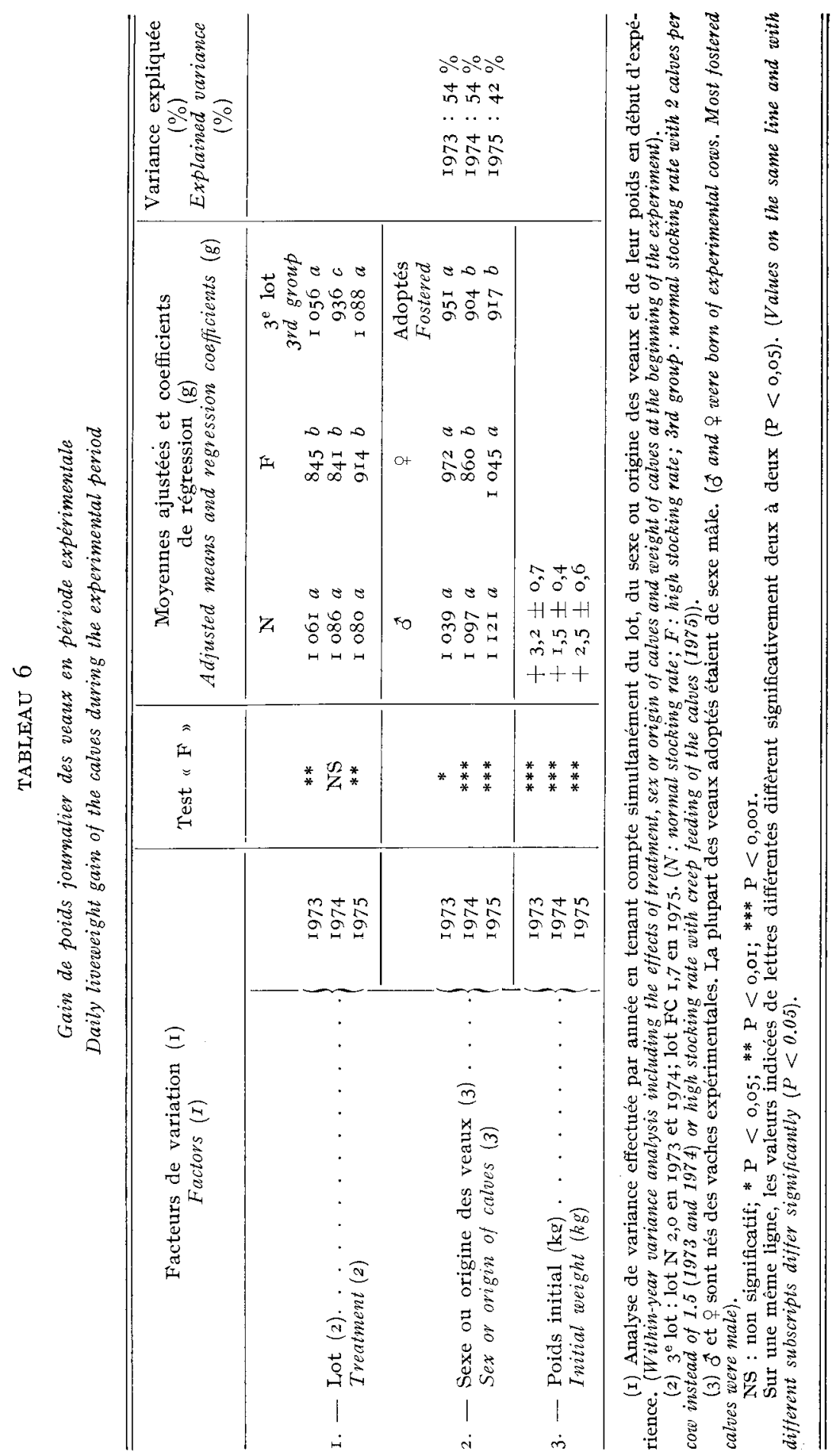


significativement $(\mathrm{P}<\mathrm{O}, \mathrm{OOI})$ avec le poids des veaux en début d'expérience de $I_{5}$ à $32 \mathrm{~g}$ pour une différence de $\mathrm{Io} \mathrm{kg}$ au début du $3^{\mathbf{e}}$ cycle et selon les années (tab1. 6). Mais cette influence du poids initial a diminué quand on avançait dans la saison de pâturage.

L,es gains de poids des veaux adoptés, tous de sexe mâle, ont été inférieurs à ceux des veaux mâles nés dans le troupeau, significativement en I974 et 1975 (一 I 8 p. IOO; $\mathrm{P}<\mathrm{O}, \mathrm{OI}$ ). Cela tient à une réussite insuffisante à l'adoption (LE Neindre, Garei et Petit, i978). Les veaux femelles ont eu un gain de poids intermédiaire en I973 et I975, mais plus faible en I974.

Pour un même poids en début d'expérience et un même sexe ou origine, les veaux du chargement fort ont eu chaque année un croît significativement plus faible que celui des veaux du chargement normal en période expérimentale : -20 p. Ioo en I973 et -23 p. Ioo en 1974 (P<0,00I), - I5 p. 100 en I975 $(\mathrm{P}<0$,or). L'apport total de $\mathrm{I} 59 \mathrm{~kg}$ d'aliment concentré par veau en I975 (lot FC I,7) a permis de combler cet handicap quasi-totalement au $3^{\mathrm{e}}$ cycle, puis totalement aux $4^{\text {e }}$ et $5^{\mathrm{e}}$ cycles. La quantité d'aliment concentré consommée par jour a augmenté tout au long de la saison et a permis aux veaux de pallier le déficit croissant en herbe. Elle est passée de $607 \mathrm{~g} / \mathrm{j}$ au $3^{\mathrm{e}}$ à I $203 \mathrm{~g}$ au $4^{\mathrm{e}}$, puis I $559 \mathrm{~g}$ au $5^{\mathrm{e}}$ cycle.

Les veaux des lots $\mathrm{N}_{2}, 0$ ont eu un gain de poids vif en expérience significativement supérieur à celui des veaux des lots $\mathrm{F}$ I,5 $(\mathrm{P}<0,001$ en I973, $\mathrm{P}<0,05$ en I974). Leur croissance a été voisine de celle des veaux du lot $\mathrm{N}_{\text {I,5 }}$ en I973, année où le chargement en vaches était plus faible de ro p. roo pour le lot $\mathrm{N} 2,0$. Elle a été inférieure de I4 P. IOO en I974 ( $\mathrm{P}<0,0 \mathrm{I})$, où les veaux pesaient plus de $r 80 \mathrm{~kg}$ en début d'expérience, ce qui les rendaient sans doute sensibles plus tôt en saison à une réduction de la quantité d'herbe offerte.

Dans tous les lots, le gain de poids journalier a baissé lors du $4^{\text {e }}$ cycle en $I 973$ et au $5^{\mathrm{e}}$ cycle en I975. Cette diminution est d'autant plus nette que le chargement en vaches ou veaux était élevé (fig. 2) et reflète sans doute la baisse de la quantité et de la qualité de l'herbe offerte. C'est vraisemblablement pourquoi les différences de croissance entre sexes ou origine des veaux ont diminué au cours de la saison de pâturage.

En chargement élevé, les gains de poids vif des veaux à l'hectare (nombre de jours de pâturage à l'hectare $\times$ gain de poids journalier individuel moyen)

TABLEAU 7

Gains de poids vif à l'hectare $(\mathrm{kg})$

Liveweight gains per hectare $(\mathrm{kg})$

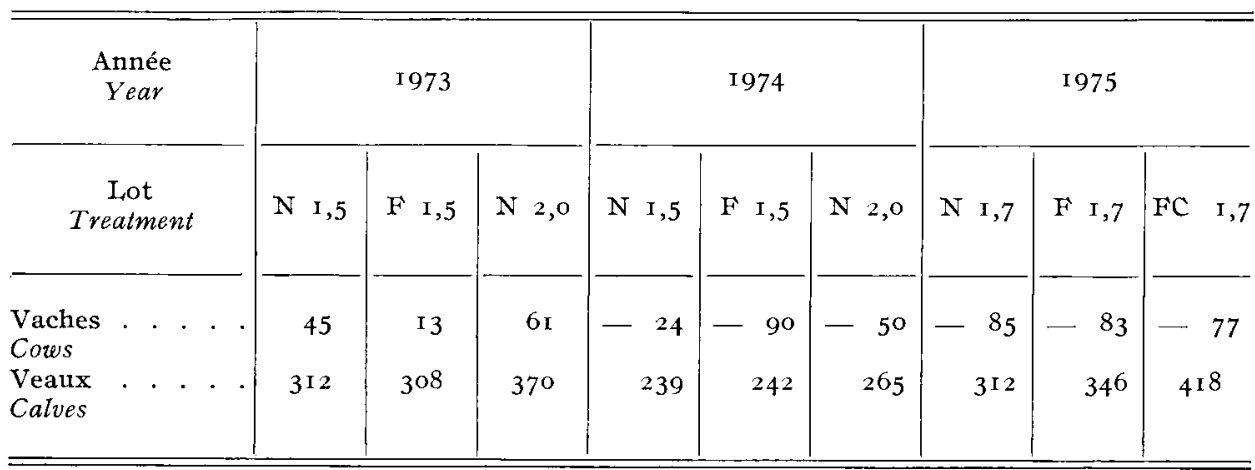


ont été équivalents à ceux obtenus dans le cas d'un chargement normal, du fait de la diminution importante des gains de poids journaliers. En revanche, le gain de poids vif des vaches à l'hectare a diminué lorsqu'augmentait le chargement en 1973 et I974 (tab1. 7).

Le gain de poids vif des veaux par hectare a été maximum pour les lots de vaches allaitant 2 veaux. Il a été accru de I9 p. Ioo en I973 et de II p. Ioo en 1974 par rapport aux lots N I,5. La réduction des gains de poids individuels en I974 a donc été largement compensée par l'augmentation du nombre de veaux allaités. Enfin, la complémentation des veaux a accru le gain de poids vif des veaux à l'hectare en $1975 \mathrm{de} 7^{2} \mathrm{~kg}$, soit $2 \mathrm{I}$ p. Ioo par rapport à celui des veaux non complémentés du lot conduit à même chargement.

\section{Discussion}

Cette étude n'a pas été conçue pour déterminer le niveau optimum de chargement, ce qui aurait nécessité de comparer plusieurs niveaux, mais simplement pour mesurer les conséquences possibles d'une augmentation importante $(+30$ p. IOo $)$ de ce chargement à partir du milieu de l'été.

I,e chargement considéré comme référence, compris entre 1,38 et 1,65 vache $(2,35$ et 2,48 veaux) à 1 'hectare, selon les années, a permis d'atteindre une croissance moyenne des veaux proche de l'objectif fixé à I Ioo $\mathrm{g} / \mathrm{j}$.

I e type de troupeau utilisé dans cette étude (vaches normandes allaitant $\mathrm{I}, 5$ à $I, 7$ veaux) devait faciliter la mise en évidence des effets du chargement sur la production laitière, souvent supérieure à Io $\mathrm{kg} /$ vache $/ \mathrm{j}$. Cependant, les vaches de race laitière allaitant plusieurs veaux ont un comportement très variable vis-à-vis des veaux étrangers supplémentaires (LE NEINDRE et GAREL, I977). Il a donc été impossible de tenir compte de la production laitière des nourrices dans l'analyse de la variabilité de la croissance de leurs veaux.

Néanmoins, les résultats montrent clairement chaque année qu'un chargement supérieur de $30 \mathrm{p}$. I 00 à la normale réduit la croissance des veaux et n'arréliore pas la production à l'hectare. Il diminue parfois significativement la production laitière moyenne des nourrices (r973 et I974). Cependant, la quantité et/ou la qualité de l'herbe offerte doit constituer le facteur essentiel limitant le gain de poids des veaux en chargement élevé, particulièrement en I975. LANGLANDS (r977) observe d'ailleurs sur agneaux une diminution significative de la quantité de matière organique ingérée sous forme d'herbe lorsqu'augmente le chargement en brebis.

C'est à la fin de la saison de pâturage que l'augmentation du chargement réduit le plus le gain de poids des veaux : les effets négatifs du chargement élevé sur la quantité d'herbe disponible se cumulent d'un cycle à l'autre, alors que les besoins en herbe des veaux vont croissant avec l'âge. Ira compétition entre vaches et veaux s'en trouve accrue. Cela a déjà été mis en évidence par DRENNAN (I97I $b$ ) qui a comparé 3 niveaux de chargement pour des vaches allaitant un seul veau $(2,4 \mathrm{I}$ (I); 3,7I (2) et 4,94 (3) vaches /ha) : à partir du début du mois d'août, dans les conditions irlandaises, il s'avérait impossible de maintenir un chargement élevé ( 2 ou 3) sans observer une chute de croissance des veaux. Mais ces chargements élevés étaient appliqués tout au cours de la saison de pâturage sans accroissement des surfaces à l'automne. De plus, bien que le troupeau de vaches allaitant 
un seul veau soit moins sensible à un accroissement du chargement que le troupeau de vaches allaitant plusieurs veaux, l'augmentation relative de chargement d'un lot à l'autre $(+50$ et + Ioo $\mathrm{p}$. Ioo) était beaucoup plus importante que dans notre étude $(+30 \mathrm{p}$. IOo).

Les résultats obtenus en 1975 indiquent que les effets sur la croissance des veaux d'une élévation de $30 \mathrm{p}$. I oo du chargement par rapport à la normale peuvent être totalement compensés par l'apport d'aliment concentré. Cependant, la quantité d'aliment concentré consommée par veau ( $160 \mathrm{~kg}$ ) a été importante. Elle a représenté I $\mathrm{kg}$ de M.S. d'aliment concentré, environ $3800 \mathrm{Kcal} / \mathrm{j}$ d'énergie métabolisable ingérée, soit 30 p. Ioo de l'énergie métabolisable ingérée sous forme d'herbe par des veaux ayant $11 n$ âge et $11 n$ gain de poids similaires (LE NEINDRE, PETIT et MULLER, I976).

L'influence du chargement sur la reprise de poids des vaches durant la totalité de la période expérimentale est beaucoup moins nette que sur le gain de poids des veaux et beaucoup plus variable entre années, bien qu'elles aient toujours disposé de moins d'herbe en chargement élevé. DRENNAN (I97I a) ne constatait de réduction toujours significative du gain de poids des vaches qu'à chargement très élevé, supérieur de roo p. Ioo à la normale. Sans doute peut-on mettre en cause, en premier lieu, les difficultés de mesure des variations de poids chez les bovins adultes. La variation totale de poids vif des vaches est faible, comparée aux variations possibles de leur important contenu digestif. Il en résulte non seulement une grande hétérogénéité des gains de poids individuels, mais il devient aussi très difficile de comparer les poids vifs des vaches qui ne pâturent pas dans des conditions équivalentes, particulièrement à la fin de la saison d'herbe. C'est pourquoi leurs gains de poids ont été calculés jusqu'à la pesée post-expérimentale effectuée en stabulation dans des conditions d'alimentation plus homogènes entre lots (I973 et I974).

En second lieu, il est possible que la vache adulte soit moins exigeante que le jeune veau sur la qualité de l'herbe offerte. Dans ce cas, l'augmentation de chargement entraînerait chez l'adulte une moindre diminution de la quantité d'herbe ingérée, particulièrement en fin de séjour sur les parcelles.

Quoiqu'il en soit, l'évolution pondérale des vaches ne reflète pas celle de leurs réserves corporelles. L'état des vaches à l'automne n'a pas été apprécié dans cette étude en raison des difficultés de sa mesure objective. Pourtant, l'état des réserves à l'automne conditionne les possibilités de restriction alimentaire durant 1'hiver suivant. Les effets du chargement sur le poids des vaches en fin de période de pâturage devraient être étudiés à plus long terme en tenant compte en particulier du niveau d'alimentation hivernal et des performances ultérieures de reproduction. L'augmentation du nombre de veaux allaités par vache au pâturage pose d'ailleurs à longue échéance les mêmes problèmes (PE'Tír, GarEL. et LE NEINDRE, I978). Dans tous les cas, il convient cependant de laisser au troupeau la possibilité de pâturer à volonté au printemps, époque où les vaches allaitantes vêlant en fin d'hiver ont leur production laitière maximum, effectuent l'essentiel de leur reprise de poids et doivent être fécondées.

L'augmentation du nombre de veaux allaités par vache pourrait en première approximation être assimilée à un accroissement du chargement en veaux, lorsque le chargement en vaches est strictement identique (année I974). Dans ce cas, les veaux du lot $\mathrm{N}_{2}, 0$ ont eu une croissance journalière inférieure de I $_{5} \mathrm{o} \mathrm{g}$ à ceux du lot $\mathrm{N}$ I,5. Bien que la production laitière des vaches ait augmenté avec le nombre de veaux allaités, la quantité de lait bue quotidiennement par veau est passée de $7, \mathrm{I} \mathrm{kg}$ (I,5 veau/vache) à $6,2 \mathrm{~kg}$ (2 veaux/vache). Si on estime à 
9o g environ l'augmentation de gain de poids par kg de lait supplémentaire (LE Neindre, Petit et Mullek, I976), l'écart observé de I50 g provient pour parties sensiblement égales des différences de quantité de lait bue et de quantité d'herbe ingérée par le veau. DRENnan ( I97 I b) observait aussi une réduction du gain de poids journalier en cas de double allaitement. Cependant, comme dans la présente étude, l'augmentation du nombre de veaux allaités par vache accroissait la production de veaux à l'hectare.

On peut enfin tenter de comparer la quantité de matière sèche offerte à une estimation grossière de la quantité d'herbe nécessaire par couple mère-veaul. $\mathrm{Si}$ on suppose que les vaches satisfont leurs besoins d'entretien (5 UFL) et de production laitière $(0,43$ UFL $/ \mathrm{kg}$ de lait $)$, que l'herbe pâturée a une valeur énergétique de 0,90 UFL $/ \mathrm{kg}$ de matière sèche (MS), elles consommeraient Io,6 $\mathrm{kg}$ de MS lorsqu'elles allaitent I, 5 veau (I0,5 $\mathrm{kg} / \mathrm{j}$ de 1ait) et II, $3 \mathrm{~kg}$ de MS 1orsqu'elles allaitent 2 veaux ( $12 \mathrm{~kg} / \mathrm{j}$ de lait). Si la quantité de MS d’herbe ingérée quotidiennement par les veaux au pâturage est proche de celle ingérée par des veaux d'âge et de poids comparables recevant de 1 'herbe coupée à volonté, elle pourrait être estimée à $\mathrm{MS}(\mathrm{kg})=4,89-0, \mathrm{I} 3 \times$ lait bu $(\mathrm{kg})$ (LE NEINDrE, résultats non publiés); elle passerait de 6,0 pour $\mathrm{I}, 5$ veau à $8,2 \mathrm{~kg}$ de MS pour 2 veaux. L'ensemble vache $+\mathrm{I}, 5$ veau serait donc capable d'ingérer $16,6 \mathrm{~kg}$ de MS d'herbe, et l'ensemble vache $+2,0$ veaux $19,5 \mathrm{~kg}$ de MS par jour.

Les vaches et veaux en chargement élevé doivent évidemment utiliser une plus grande proportion de l'herbe offerte (I06 p. I00 en I974 et 68 p. Ioo en I975) que celles en chargement normal (72 et 54 p. Ioo). De même, le troupeau de vaches à 2 veaux doit utiliser en $197482 \mathrm{p}$. Ioo de $1^{\prime}$ herbe offerte au lieu de $72 \mathrm{p}$. Ioo dans le cas du troupeau à $I, 5$ veau. Ces valeurs sont évidemment grossières puisqu'elles ne tiennent pas compte, entre autres, des difficultés d'appréciation de la quantité d'herbe présente, de la pousse de l'herbe pendant le temps de présence des troupeaux, de la proportion d'herbe inconsommable, de l'accroissement des besoins des animaux dans les conditions du pâturage ni des variations du poids vif des vaches. Elles montrent toutefois que, pour obtenir une croissance des veaux élevée, il convient d'offrir une quantité d'herbe souvent très nettement supérieure aux besoins du troupeau. Si on admet que les vaches sont capables d'utiliser une plus grande proportion de 1'herbe offerte sans réduire sensiblement 1a reconstitution de leurs réserves corporelles, il devient alors préférable de fournir une alimentation complémentaire aux veaux en automne.

\section{Conclusion}

Par rapport à un chargement normal permettant un gain de poids des veaux d'environ I I $00 \mathrm{~g} / \mathrm{j}$, une augmentation de $30 \mathrm{p}$. Ioo du chargement en vaches allaitantes (I,97 au lieu de I,53 vache/ha) à partir du début de l'été provoque une diminution sensible de ce gain de poids de $200 \mathrm{~g}$ en moyenne. Cette réduction de croissance s'accentue en fin de saison et ne permet pas d'accroître la production à l'hectare. La forte exigence des veaux vis-à-vis de la qualité du pâturage ne serait pas compatible avec un tel niveau de chargement. Néanmoins, un apport de $\mathbf{r} 60 \mathrm{~kg}$ d'aliment concentré aux veaux compense l'effet de l'augmentation du chargement.

D'autre part, à même niveau de chargement, l'augmentation du nombre de veaux allaités par vache $(2,0$ au lieu de $I, 5$ veau par vache normande) réduit les 
gains de poids vif des veaux (de I5o g en moyenne), mais accroît toutefois la production à l'hectare. Cette réduction de croissance est imputable à une diminution de la quantité de lait bue mais aussi à la simple augmentation du chargement en veaux. Cet ensemble de résultats confirme l'importance de la qualité du pâturage des veaux allaités par leur mère, particulièrement en automne.

La production laitière des vaches allaitantes subit peu l'influence du chargement au pâturage, dans les limites des chargements et avec le type de vaches utilisé dans cette étude.

Enfin, les gains de poids des vaches semblent être moins sensibles que ceux des veaux à l'augmentation du chargement. Cela résulte toutefois en partie des variations importantes de leur contenu digestif. Les effets sur les vaches de l'accroissement du niveau de chargement devraient être étudiés à plus long terme et tenir compte des possibilités de restrictions alimentaires en hiver. Ils devraient aussi être étudiés dans diverses conditions de pâturage et avec différents types de vaches.

Accepté pour publication en août 1980.

\author{
Summary \\ Suckling cows at pasture: \\ effect of stocking rate in summer and autumn
}

At the Pin-au-Haras experimental farm in Normandy two stocking rates were compared on grazing suckling cows, from July to October, for 3 successive years. Dairy cows (Norman breed) which suckled I.5 (I973-I974) or I.7 (I975) calves per cow were used. Normal stocking rates (N I.5 or N I.7) should allow a daily growth rate of the calves up to I.I $\mathrm{kg}$. High stocking rates (F I.5 or F I.7) were 30 p. I oo higher and obtained by using additional cows and calves (table $\mathbf{I}$ ).

A third treatment was added each year: either a normal stocking rate with 2.0 calves per cow instead of $1.5\left(\mathrm{~N}_{2.0}\right)$ in 1973 and I974, or a high stocking rate which consisted of creep feeding the calves with concentrates ( $\mathrm{FC}$ I.7), in 1975 .

The experimental period started at the beginning of summer and stopped at the end of the normal grazing season, the end of October. In spring, enough grass is generally available when the cows have to recover body reserves and be mated. Therefore normal grazing pressure was used for all animals till early July.

The experimental area, previously grazed during 2 cycles or cut for silage, was divided into 3 balanced groups of 5 to 6 paddocks. Each group was assigned to one herd. The three herds moved simultaneously through a rotational grazing system. The herbage mass in each paddock was measured just before the herds started to graze. The animals were weighed once per grazing cycle and the milk production of the cows was estimated by weighing the calves before and after suckling. Within-year variance-covariance anlalyses were made on: I) total liveweight gain of cows from start of experiment up to either last weighing on grass feeding or first weighing on winter feeding; 2) daily liveweight gain of the calves during each cycle and during the entire experimental period; 3) milk production of the cows during experiment.

Initial characteristics of animals are summarized in table 2, and grazing process in table 3 . As grazing season went on, herbage mass per ha and stocking rate decreased but daily herbage allowance per cow increased in $\mathrm{N}$-treatments. Herbage mass per ha was lowest in $\mathrm{F}$ treatment. Daily herbage allowance per F-cow was 68 and $79 \mathrm{p}$. I oo of daily herbage allowance per N-cow in 1974 and 1975 , respectively.

Grazing cows in N 1.5-treatment recovered more (or lost less) liveweight in I973 and I974 than did F r.5-cows, but the significance of differences disappeared when liveweight gains were calculated up to the start of winter feeding (table 4 and fig. 2). In I975, N I.7-cows lost more weight than F I.7-cows but not significantly after correction for estimated weights of intrauterine contents. Cows suckling two calves (N 2.o-cows) lost more weight than $\mathrm{N}$ I.5-cows 
in 1974 when designed relative stocking rate was effective, but gained more weight in 1973 because of the low relative stocking rate (table 3 ).

$\mathrm{N}$ 2.0-cow produced significantly more milk than did N I.5-cows (table 5). Increasing the stocking rate significantly decreased the milk production of the nursing cows in I973 and I974 but not in I975.

Average daily liveweight gain (ADG) in the $\mathrm{N}$-calves was close to expected liveweight gain (I.I kg/day) (table 6). ADG increased significantly with initial liveweight. ADG of the fostered male calves was lower than ADG of males born from the herds (significantly in I974 and 1975). After correction for initial liveweight and origins of calves, increasing the stocking rate significantly reduced $\mathrm{ADG}$ of calves. The differences increased during the grazing season (fig. 2) while the quantity and quality of herbage probably decreased. Adjusted means of ADG, averaged for the 3 years, were $\mathrm{I} 076$ and $867 \mathrm{~g}$ for $\mathrm{N}$ - and $\mathrm{F}$-calves respectively. Creep-fed calves (FC I.7) ate I60 kg of concentrates during the entire experimental period and had ADG similar to N I.7 calves, despite a higher stocking rate. With the same relative stocking rate of cows in 1974, ADG was significantly lower in $\mathrm{N} 2.0$ calves than in N I.5 calves (- I $50 \mathrm{~g} / \mathrm{d})$ because of a lower milk intake per calf and probably because of the higher stocking rate of calves.

Calf liveweight gain per ha was about the same for $N_{1.5}$ and F I.5 treatments, but was maximum for $\mathrm{N} 2.0$ treatment in 1973 and 1974 , and for $F C$ I.7 treatment in 1975 (table 7).

These data clearly demonstrate that, under good grazing conditions allowing winter born calves to have liveweight gains up to $I$. I kg/day at normal stocking rate, the growth rate of the calves drops $20 \mathrm{p}$. roo when the stocking rate of nursing cows is increased by 30 p. roo in sumner and autumn grazing. These results stress the importance of diet selection by the grazing suckled calves. Negative effects of high stocking rate can be compensated by creep feeding the calves. At a normal stocking rate, increasing the number of calves suckled per nursing cow decreases the calves' growth rate but increases the calf liveweight gain per ha. Cows seem to be less sensitive to stocking rate than their calves which partly results from difficulties in determining liveweight and body composition changes of adult animals in grazing experiments. The effects of stocking rate on grazing suckling cows have to be studied with different types of cows under various grazing conditions. Possibilities of low feeding levels during the following winter in long term experiments should be considered.

\section{Références bibliographiques}

BERESKIN B., TOUCHBERIY R, W., I967. Some effects of pregnancy on body weight and paunch girth. J. Dairy Sci., 50, 220-224.

DRENNAN M. J., I97 I a. Single-suckled beef production : I. - Influence of stocking rate during the grazing season, creep grazing of the calf and double suckling on live weight changes and milk production of the cows. Ir. J. Agric. Res., 10, $287-295$.

Drennan M. J., I97 I $b$. Single-suckled beef production : II. - Influence of stocking rate during the grazing season, creep grazing of the calf and double suckling on calf performance. $I \gamma . J$. Agric. Res., 10, 297-305.

GibB M. J., Treacher T. T., I978. The effect of herbage allowance on herbage intake and performance of ewes and their twin lambs grazing perennial ryegrass. $J$. Agric. Sci., 90, I 39147.

GRENET N., r974. Exemple d'utilisation de vaches laitières comme mères allaitartes. In " $L$ 'Exploitation des troupeaux de vaches allaitantes ", Suppl. Bull. Techn. C.R.Z.V. Theix, I.N.R.A., 307-32I.

Harts F. J., I97o. Multiple suckling of calves. $J$. Ir. Grassl. Anim. Prod. Assoc., 6, $65^{-78}$.

Henessy D. W., Robinson G. G., I979. The herbage intake, eating behaviour and calf production of beef cows grazing improved pastures on the Northern Table lands of New South Wales. Aust. J. Exp. Agric. Anim. Husb., 19, 2 I6-268.

LANGLANDS J. P., I977. The intake and production of lactating Merino ewes and their lambs grazed at different stocking rates. Aust. J. Agric. Res., 28, I33-142.

LE DU Y. L. P., BARker J. M., BAkER R. D., I976. Milk fed calves. 2. - The effect of length of milk feeding period and milk intake upon herbage intake and performance of grazing calves. J. Agric. Sci., 87, 197-204.

LE NEINDRE P., PETIT M., MULLFR A., I 976. Quantités d'herbe et de lait consommées par des veaux au pis. Ann. Zootech., 25, 52 I-53I . 
Le Neindre P., Garel J. P., I977. Étude des relations mère-jeune chez les bovins domestiques : comparaisons des liaisons existant entre la mère et des veaux légitimes ou adoptés. Biol. Behav., 2, 39-49.

Le Neindre P., Garec J. P., Petit M., i978. Allaitement de deux veaux par des vaches de race Salers : 2. - Étude de l'adoption. Ann. Zootech., 27, 553-569.

Pe'Ti'T M., Gareí J. P., LE NeINDRE P., 1978. Allaitement de deux veaux par des vaches de race Salers : I. - Productions comparées de vaches allaitant I ou 2 veaux. Ann. Zootech., 27, 533-55I.

SEEBECK R. M., I973. The effect of body weight loss on the composition of Brahman cross and Africander cross steers. I. - Empty body weight, dressed carcass weight and offal components. J. Agric. Sci., 80, 201-2 ro. 